Ahmed El-Rafei, Tobias Engelhorn, Simone Waerntges, Arnd Doerfler, Joachim Hornegger and Georg Michelson: Automatic Segmentation of the Optic Radiation Using DTI in Healthy Subjects and Patients with Glaucoma. Computational Vision and Medical Image Processing - Recent Trends, Computational Methods in Applied Sciences, 2011, Volume 19, 1-15, DOI: 10.1007/978-94-007-0011-6_1

This is the author submitted version of an article whose final and definitive form has been published in Computational Vision and Medical Image Processing - Recent Trends, Computational Methods in Applied Sciences (c) 2011 Springer. The original publication is available at www.springerlink.com with DOI: 10.1007/978-94-007-0011-6_1. 


\title{
Automatic Segmentation of the Optic Radiation Using DTI in Healthy Subjects and Patients with Glaucoma
}

\author{
Ahmed El-Rafei, Tobias Engelhorn, Simone Waerntges, Arnd Doerfler, Joachim \\ Hornegger, and Georg Michelson
}

\begin{abstract}
The complexity of the diffusion tensor imaging (DTI) data and the interpersonal variability of the brain fiber structure make the identification of the fibers a difficult and time consuming task. In this work, an automated segmentation system of the optic radiation using DTI is proposed. The system is applicable to normal subjects and glaucoma patients. It is intended to aid future glaucoma studies. The automation of the system is based on physiological and anatomical information to produce robust initial estimates of the optic radiation. The estimated optic radiation initializes a statistical level set framework. The optic radiation is segmented by the surface evolution of the level set function. The system is tested using eighteen DTI-datasets of glaucoma patients and normal subjects. The segmentation results were compared to the manual segmentation performed by a physician experienced in neuroimaging and found to be in agreement with the known anatomy with $83 \%$ accuracy. The automation eliminates the necessity of medical experts' intervention and facilitates studies with large number of subjects.
\end{abstract}

Ahmed El-Rafei and Joachim Hornegger

Pattern Recognition Lab, Department of Computer Science,

and Erlangen Graduate School in Advanced Optical Technologies (SAOT),

Friedrich-Alexander University Erlangen-Nuremberg, Germany

e-mail: ahmed.el-rafei@informatik.uni-erlangen.de, joachim.hornegger@informatik.uni-

erlangen.de

Tobias Engelhorn and Arnd Doerfler

Department of Neuroradiology, Friedrich-Alexander University Erlangen-Nuremberg, Germany e-mail: Tobias.Engelhorn@uk-erlangen.de, Arnd.Doerfler@uk-erlangen.de

Simone Waerntges

Department of Ophthalmology, Friedrich-Alexander University Erlangen-Nuremberg, Germany

e-mail: Simone.Waerntges@uk-erlangen.de

Georg Michelson

Department of Ophthalmology,

and Interdisciplinary Center of Ophthalmic Preventive Medicine and Imaging,

Friedrich-Alexander University Erlangen-Nuremberg, Germany

e-mail: Georg.Michelson@uk-erlangen.de 


\section{Introduction}

Glaucoma is the second leading cause of blindness in the world. The damage caused by glaucoma is irreversible. The progression of glaucoma can be delayed significantly if glaucoma is detected in early stages. Therefore, methods for screening, early diagnosis and better understanding of glaucoma and its progression are needed.

Most of the existing eye imaging modalities focus on imaging the eye in general and the retina in particular. Many studies were performed to investigate the correlation between glaucoma and retinal changes such as retinal nerve fiber atrophy, retinal vessels, and optic disk changes $[24,16,33]$. The human visual system does not only consist of the eye but it extends through the optic nerve into the brain till it reaches the visual cortex. The visual pathway consists of four neurons. The first neuron (photoreceptors) and the second neuron (amacrine and bipolar cells) lie within the retina in the eye. The third neuron (retinal ganglion cells) connects the retina with the brain. The axons of the third neuron leave the eye and end in the lateral geniculate nucleus where the fourth neuron begins. The axons of the fourth neuron neuronal cells carry the visual information and end up in the visual cortex V1. The intracerebral part of the fourth neuron is called the optic radiation. Correlation has been shown between glaucoma and parts of the visual system such as the optic nerve and the optic radiation $[18,13]$. Nevertheless, the effect of glaucoma on the visual system is not fully addressed yet.

In this work we aim to provide a system for the automatic identification of the optic radiation in normal subjects and glaucoma patients. DTI is used to segment the optic radiation as it is the only imaging modality that allows for the identification of white matter fiber structure non-invasively. This is a step towards a better understanding of the changes caused by glaucoma in this part of the human visual system.

In the last two decades, diffusion tensor imaging has received a lot of attention due to its clinical applications [39, 11]. Diffusion weighted imaging (DWI) is proven to be effective in the early diagnosis and investigation of cerebral diseases such as acute stroke [28, 21] and abscesses [7]. Diffusion tensor derived parameters such as the degree of anisotropy and the diffusivity parameters are used to evaluate certain neural pathologies and were found to be sensitive to white matter abnormalities. Axonal degeneration evaluated by diffusion tensor derived parameters were evident in the temporal lobe for mild cognitive impairment and Alzheimer disease patients $[8,17]$. In relapsing-remitting multiple sclerosis, reduced anisotropy accompanied by increased isotropic apparent diffusion were observed correlating to the signature of Wallerian degeneration [15]. The process of normal human brain maturation and aging affecting the structure of myelin were monitored using DTI [34, 19]. Furthermore, DTI is the only imaging modality that allows tracking the white matter fibers in-vivo and non-invasively [3, 29], and it enables the construction of an atlas of white matter fibers in the human brain [36, 27].

As the basis of the DTI, diffusion weighted imaging (DWI) is based on magnetic resonance signal attenuation due to restricted diffusion of water molecules along the 
diffusion weighting gradient field $[23,26]$. The cell membranes and myelin sheaths surrounding the axons act as a guided tube for the diffusion process within the axons. Thus, they limit the diffusion in the direction perpendicular to the axons while increasing the average diffusion along the axons resulting in a highly anisotropic diffusion. The diffusion of water like molecules can be used to identify fiber orientation which coincide with the average diffusion direction and so for to depict the microstructure of the brain white matter. The diffusion tensor relies on modeling the diffusion process within a specified volume by a Gaussian process with a zero mean. The tensor corresponds to the inverse of the covariance matrix of the diffusion process and is calculated from the diffusion weighted images. Analyzing the diffusion tensor gives significant diffusion related information such as the main diffusion direction within the specified volume and the degree of anisotropy. This information is used to identify the white matter structure within the brain.

Many algorithms were proposed for the identification of white matter tracts using DTI. The dominant category is tractography which is based on following the fiber tracts using the principal diffusion direction [9, 4, 35, 22]. Tractography suffers from accumulated tracking errors during the tracking process. Connectivity maps were suggested $[38,30,20]$ to explore the probability of connectivity between a selected seed point and the surrounding neighborhood which can be the whole brain. Connectivity maps have the main disadvantage that they do not provide a straightforward plausible visualization of the results. The split and merge technique [6] attempts to avoid the accumulated errors of tractography by identifying short tracts. This is done by limiting the tracking process to a certain number of steps. Then it provides a degree of membership of the extracted tracts belonging to the same fiber. The practicality of the split and merge technique is limited because it does not describe the complete fiber pathway. Segmentation approaches of DTI [37, 40, 14] are more suitable for identifying coherent densely packed bundles of axons. The segmentation avoids the drawbacks from both connectivity maps and tractography such as tracking accumulation errors and the need to merge the individual tracts to obtain fiber bundles. Furthermore, it relies on the coherency within the fiber bundle of interest. Therefore, the segmentation approach is adopted in this work.

Most of the proposed white matter identification algorithms did not address the problem of algorithm initialization. They rely on the interaction of medical experts to select the seed points or the region of interest of the desired fiber tracts in tractography algorithms or the initialization of the segmentation engines to include the desired fiber bundle. This is a rather time consuming process and might limit the number of subjects in clinical studies that involves DTI. The proposed segmentation system utilizes the physiological properties of the optic radiation to produce a robust initialization of the proposed segmentation system in both healthy and pathological subjects with glaucoma.

The proposed segmentation system utilizes the complete tensor information in a statistical level set frame work that takes into account the Riemannian nature of the tensor space. It consists of the following steps: First the diffusion tensor and related anisotropy measures are calculated from the diffusion weighted images. The calculated diffusion tensor data is transformed into the Log-Euclidean framework and 
interpolated as presented in Sect. 2. In Sect. 3, DTI-data is regularized to increase the coherency of the optic radiation fiber bundle before obtaining an initial estimate of the optic radiation using thresholding and connectivity analysis. The midbrain is initially identified using a similar analysis to that of the optic radiation. The system extends the statistical level set framework for DTI segmentation developed by Lenglet et al. [25] to be used in conjunction with the Log-Euclidean dissimilarity distance as detailed in Sect. 4. The optic radiation is obtained by iteratively evolving the level set function. Finally, the output from the level set framework is adjusted based on the relative location of the optic radiation and the midbrain. Section 5 contains the results and discussion. The conclusion and future work are stated in Sect. 6 .

\section{Interpolation in the Space of Diffusion Tensors}

The diffusion tensors are $3 \times 3$ symmetric positive definite matrices. The space of diffusion tensors is a convex subset of the vector space $\mathbb{R}^{(3)^{2}}$ and does not form a vector space using a Euclidean metric $[12,31]$. Thus, the decomposition of the diffusion tensors could result in non-physical negative eigenvalues. Moreover, the Euclidean framework is not appropriate for dealing with tensors because the swelling effect where the average of diffusion tensors with the same determinant could result in a mean tensor with a larger determinant [10]. Thus, the Riemannian nature of the tensor space should be taken into account when handling the diffusion tensors.

Dissimilarity metrics has been proposed to overcome the limitations of the Euclidean framework. An information theoretic measure called the J-divergence is proposed [37] based on the symmetric Kullback-Leibler divergence between two Gaussian probability densities. The J-divergence distance between two diffusion tensors is given by Eq. 1 and is affine-invariant. i.e. the distance between tensors are independent from affine transformation of the coordinate system.

$$
d_{J}\left(\mathrm{DT}_{1}, \mathrm{DT}_{2}\right)=\frac{1}{2} \sqrt{\operatorname{tr}\left(\mathrm{DT}_{1}^{-1} \mathrm{DT}_{2}+\mathrm{DT}_{2}^{-1} \mathrm{DT}_{1}\right)-2 n}
$$

where $\operatorname{tr}($.$) is the matrix trace operator, \mathrm{n}$ is the size of the diffusion tensors $\mathrm{DT}_{1}$ and $\mathrm{DT}_{2}$.

Fletcher and Joshi [12] deal with the space of diffusion tensors as a curved manifold called Riemannian symmetric space. They derived a Riemannian metric on the space of diffusion tensors. The proposed metric accounts for the positive definiteness constraint ensuring that the eigenvalues of the diffusion tensors are positive.

The Log-Euclidean framework proposed by Arsigny et al. [2] provides a Riemannian framework to deal with the diffusion tensors. Using this framework, the diffusion tensor space of positive semi definite matrices can be transformed into the space of symmetric matrices, i.e. a vector space. Additionally, all operations performed on vectors can be used on the vector form of the diffusion tensor in the Log-Euclidean framework. Despite the similar properties of the Log-Euclidean met- 
ric compared to other dissimilarity distances such as the J-divergence distance or the Riemannian metric by Fletcher and Joshi, the Log-Euclidean framework has a similar behavior but at a significantly lower computational cost as it involves vector operations.

The Log-Euclidean distance $d_{L E}$ between tensors $\mathrm{DT}_{1}$ and $\mathrm{DT}_{2}$ is defined by

$$
d_{L E}\left(\mathrm{DT}_{1}, \mathrm{DT}_{2}\right)=\left\|\log \left(\mathrm{DT}_{1}\right)-\log \left(\mathrm{DT}_{2}\right)\right\|
$$

where $\log$ is the matrix logarithm.

The interpolation of the DTI-data is necessary in order to obtain a volumetric identification of the optic radiation. Interpolation of diffusion tensors in the Euclidean framework results in the non-physical swelling effect. This effect is also evident in interpolating two tensors, where it is possible to get an interpolated tensor that has a larger determinant than the original tensors. Interpolation in the LogEuclidean framework avoids the swelling effect at a computationally attractive cost. The diffusion tensor DT is interpolated trilinearly at non-grid position $x$ as the LogEuclidean weighted sum of $N$ tensors in a neighborhood of the non-grid position $x$. The weights are inversely proportional to the spatial distance between the non-grid position and the locations of the tensors in the neighborhood. The used interpolation formula is

$$
\mathrm{DT}(x)=\exp \left(\frac{\sum_{i=1}^{N} w_{i}(x) \log \left(\mathrm{DT}\left(x_{i}\right)\right)}{\sum_{i=1}^{N} w_{i}(x)}\right)
$$

where exp and log are the matrix exponential and logarithm respectively.

\section{Initial Estimation of the Optic Radiation and the Midbrain}

In this step, the optic radiation and the midbrain are initially identified. The diffusion tensor data is first regularized by applying Perona-Malik diffusion filtering [32]. Perona and Malik proposed an anisotropic diffusion filtering technique based on controlling the heat flow according to the presence of edges. The edges are estimated by the magnitude of the image gradient. The diffusivity is non-linearly inversely proportional to the magnitude of the image gradient, i.e. the diffusion is limited at large image gradients indicating the presence of an edge with high probability. Conversely, the diffusion is increased at small image gradients. The evolution of the image $f(x, y, z): \Omega \subset \mathbb{R}_{3} \rightarrow \mathbb{R}$ is governed by the following diffusion equation

$$
\frac{\partial f}{\partial t}=\operatorname{div}(\rho(\|\nabla f\|) \nabla f)
$$

where $\rho=e^{-\frac{\|\nabla f\|^{2}}{k}}$ or $\rho=\frac{1}{1+\|\nabla f\|^{2} / k}$. 
The diffusion filtering is applied to the transformed Log-Euclidean vector form of the diffusion tensors componentwise. Regularization is performed to reduce the noise and to increase the coherency inside the fiber bundles while preserving the boundaries of the fiber bundles.

The initial estimation of the optic radiation is based on the fact that the main fiber bundle of the optic radiation is dominated by diffusion in the anterior-posterior direction. Moreover, the optic radiation is a massive fiber bundle which occupies a significant part of the brain white matter. This physiological information regarding the diffusion direction and the size of the optic radiation gives a unique discrimination of the optic radiation from other fiber bundles.

The diffusion tensor is analyzed using eigen-decomposition as given by Eq. 5 to determine the principal diffusion direction (PDD) which is the eigenvector of the tensor corresponding to the largest eigenvalue. The degree of anisotropy is determined by the fractional anisotropy (FA) [5] and is calculated from the diffusion tensor eigenvalues using Eq. 6.

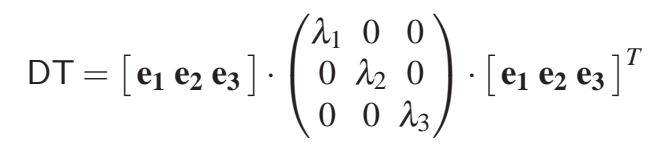

where $\mathbf{e}_{1}, \mathbf{e}_{2}$ and $\mathbf{e}_{3}$ are the diffusion tensor eigenvectors corresponding to $\lambda_{1}, \lambda_{2}$ and $\lambda_{3}$ which are the diffusion tensor eigenvalues in a descending order.

$$
F A=\sqrt{\frac{3}{2}} \frac{\sqrt{\left(\lambda_{1}-\lambda\right)^{2}+\left(\lambda_{2}-\lambda\right)^{2}+\left(\lambda_{3}-\lambda\right)^{2}}}{\sqrt{\lambda_{1}^{2}+\lambda_{2}^{2}+\lambda_{3}^{2}}}
$$

where $\lambda=\frac{\left(\lambda_{1}+\lambda_{2}+\lambda_{3}\right)}{3}$.

The image is analyzed on a voxel by voxel basis to create a binary mask representing the initial optic radiation. The vector corresponding to principal diffusion direction has three components: the anterior-posterior component (AP), the left-right component (LR) and the superior-inferior (SI) component. The three components at each voxel are compared and the foreground voxels of the binary mask are selected to have a dominant AP component. The foreground voxels satisfies the inequalities given by Eq. 7 that is the AP-component is greater than a user specified factor $\left(A P_{\text {thres }}\right)$ of the sum of the other two components and a fractional anisotropy value greater than 0.2. The fractional anisotropy threshold is used to ensure the coherency of the fiber bundle and that the partial volume effects [1] are avoided. In DTI the partial volume effects are the result of the limitation of the tensor model to describe complex fiber situations such as fiber crossing or branching situations within a voxel. This results in a reduced fractional anisotropy and a misleading principal diffusion direction. The remaining voxels that do not satisfy the selection criteria are set as the background of the binary image. 


$$
A P>A P_{\text {thres }} \times(L R+S I) \quad \text { and } \quad F A>0.2
$$

A three dimensional 6-neighborhood connectivity analysis is performed on the binarized image. Connected objects are determined and the optic radiation is initially identified as the largest object dominated by diffusion in the anterior-posterior direction. This estimation will be used in the segmentation step as an initialization of the level set.

The analysis applied to estimate the optic radiation is similarly applied to identify the midbrain. The analysis takes into account that the midbrain is characterized by diffusion in the superior-inferior direction and is located in the neighborhood of the centers of the axial brain slices. The relative position of the estimated midbrain to the optic radiation will be used in a later step to refine the segmentation of the optic radiation.

\section{Segmentation Using a Statistical Level Set Framework}

The segmentation is performed in two steps. First, the DTI is segmented using a statistical level set framework. The initially estimated optic radiation as described in Sect. 3 is used as the initial surface. Second, the results from the level set framework are adjusted based on anatomical information between the midbrain and the optic radiation.

We extend the surface evolution framework developed by Lenglet et al. [25] to work with the Log-Euclidean dissimilarity measure given in Eq. 2. In the following we present briefly the mathematical formulation of the level set framework in the case of the Log-Euclidean framework. For further details see [25, 2]. The diffusion tensor DT $(x)$ at voxel $x$ is mapped to the space of symmetric matrices and transformed into a vector form $\beta(x)$ using the following mapping:

$$
\beta(x)=\operatorname{vec}(\log (\mathrm{DT}(x)))
$$

where vec is the mapping of the $3 \times 3$ symmetric matrices to the corresponding 6-dimensional vectors.

Using the notation in Eq. 8, the mean, covariance matrix and Gaussian distribution between diffusion tensors can be defined as :

$$
\begin{gathered}
\mu_{L E}=\frac{1}{N} \sum_{i=1}^{N} \beta\left(x_{i}\right) \\
\operatorname{Cov}_{L E}=\frac{1}{N-1} \sum_{i=1}^{N}\left(\beta\left(x_{i}\right)-\mu_{L E}\right)\left(\beta\left(x_{i}\right)-\mu_{L E}\right)^{T}
\end{gathered}
$$




$$
\begin{aligned}
P_{L E}( & \left.\beta\left(x_{i}\right)\right)=\frac{1}{\sqrt{(2 \pi)^{6}\left|\operatorname{Cov}_{L E}\right|}} \\
& \times \exp \left(-\frac{\left(\beta\left(x_{i}\right)-\mu_{L E}\right)^{T} \operatorname{Cov}_{L E}^{-1}\left(\beta\left(x_{i}\right)-\mu_{L E}\right)}{2}\right)
\end{aligned}
$$

The spatial gradient of the diffusion tensor in the vector space is given by

$$
\begin{aligned}
|\nabla \beta(x)|^{2}=\frac{1}{2} \sum_{k=1}^{3} \sum_{s= \pm 1} \operatorname{tr}( & \left(\beta(x)-\beta\left(x+s \times i_{k}\right)\right) \\
& \left.\times\left(\beta(x)-\beta\left(x+s \times i_{k}\right)\right)^{T}\right)
\end{aligned}
$$

where $i_{k}, k=1,2,3$ denotes the canonical basis of $\mathbb{R}_{3} . s \in\{1,-1\}$ and denotes the forward and backward approximations of the gradient, $t r$ is the trace of a matrix.

The idea of the statistical surface evolution is to seek the optimal partitioning of the tensor image ( $\beta$ in the Log-Euclidean case) by maximizing a posteriori frame partition probability for the diffusion tensor image with image domain $\Gamma$. This is done in a level set framework, where the image is partitioned into three regions based on a level set function $\phi$ : inside $\Gamma_{i n}$, outside $\Gamma_{\text {out }}$ or on the boundary $\Gamma_{B}$. The boundary is defined as the zero-crossing of $\phi$. The probability distributions of the tensors inside $\left(p_{\text {in }}\right)$ and outside $\left(p_{\text {out }}\right)$ regions are modeled by Gaussian distributions on tensors using Eq. 11. The partition probability is given by

$$
P(\beta \mid \phi)=\prod_{x \in \Gamma_{\text {in }}} p_{\text {in }}(\beta(x)) \prod_{x \in \Gamma_{\text {out }}} p_{\text {out }}(\beta(x)) \prod_{x \in \Gamma_{B}} p_{b}(\beta(x))
$$

The boundary probability distribution $p_{b}$ is selected to have a value of approximately one for high gradients of the diffusion tensors (using Eq. 12 for gradient calculations) and a value of approximately zero for low gradients as the following relation indicates.

$$
p_{b}(\beta(x)) \propto \exp (-g(|\nabla \beta(x)|))
$$

where $g(u)=1 /\left(1+u^{2}\right)$.

This leads to the energy minimization formulation:

$$
\begin{aligned}
E & \left(\phi, \mu_{L E_{\text {in } / \text { out }}}, \operatorname{Cov}_{L E_{\text {in } / \text { out }}}\right) \\
& =v \int_{\Gamma} \delta(\phi)|\nabla \phi| d x+\int_{\Gamma} \delta(\phi)|\nabla \phi| g(|\nabla \beta(x)|) d x \\
& -\int_{\Gamma_{\text {in }}} \log \left(p_{\text {in }}(x)\right) d x-\int_{\Gamma_{\text {out }}} \log \left(p_{\text {out }}(x)\right) d x
\end{aligned}
$$

where $\delta$ is the Dirac delta function.

The following Euler-Lagrange equation is used to evolve the level set function 


$$
\begin{aligned}
\frac{\partial \phi}{\partial t}= & \delta(\phi)\left((v+g(|\nabla \beta(x)|)) \operatorname{div}\left(\frac{\nabla \phi}{|\nabla \phi|}\right)\right. \\
& \left.+\frac{\nabla \phi}{|\nabla \phi|} \cdot \nabla g(|\nabla \beta(x)|)+\log \left(\frac{p_{\text {in }}}{p_{\text {out }}}\right)\right)
\end{aligned}
$$

The level set function in Eq. 16 is evolved iteratively to obtain the desired segmentation and the statistics are updated after each iteration.

The output from the level set framework contains the fiber bundle of the optic radiation and additional bundles connected to it such as traces of the optic tract. The reason for this is that the optic tract is connected to the optic radiation and the diffusion direction is also anterior-posterior in the connection area so traces of the optic tract are segmented as well. The lateral geniculate nucleus (LGN) connects the optic radiation to the optic tract and is located laterally to the midbrain. Therefore, the LGN position can be used to separate the optic tract from the optic radiation. Based on this anatomical information, the segmented region is automatically adjusted in order to confine the segmentation results to the part representing the optic radiation. The relative position of the segmented optic radiation to the midbrain is used instead of the relative position to the LGN because the midbrain is larger, more reliable to identify and in turn more robust. The midbrain is previously identified in the initialization step. The plane corresponding to the anterior boundary of the segmented midbrain is selected as the separation level between the optic radiation and the optic tract. The segmentation results anterior to the selected plane are eliminated leaving the optic radiation and approximately eliminating the part corresponding to the optic tract.

\section{Results and Discussion}

Eighteen subjects were examined by ophthalmologists and categorized into two age matched groups. The first group represents the subjects that were diagnosed with primary open angle glaucoma and the other group represents the normal subjects. The glaucoma group contains 9 subjects with a mean \pm standard deviation age of $66 \pm 11.8$ years with 7 females and 2 males, while the normal group contains 9 subjects with a mean \pm standard deviation age of $67.1 \pm 8.1$ years with 6 females and 3 males. Further ophthalmological and neuroradiological examinations were performed and did not provide indications of microangiopathy or irregularly developed optic radiation.

The subjects were scanned using a 3T-MRI scanner. The diffusion weighted images were acquired using a single-shot, spin echo, echo planar imaging (EPI) as an imaging sequence with repetition time (TR) $3400 \mathrm{~ms}$, echo time (TE) $93 \mathrm{~ms}$, field of view (FoV) $230 \times 230 \mathrm{~mm}^{2}$, acquisition matrix size of $128 \times 128$ reconstructed to $256 \times 256$, seven signal averages, and partial Fourier acquisition of $60 \%$. The axial slices have a thickness of $5 \mathrm{~mm}$ and $1 \mathrm{~mm}$ interslice spacing. Diffusion weighting 
were applied with a maximal b-factor of $1000 \mathrm{~s} / \mathrm{mm}^{2}$ along 15 icosahedral directions complemented by one scan with $b=0$. The diffusion tensors were calculated from the measured diffusion weighted images along with fractional anisotropy, eigenvectors and eigenvalues on a voxel by voxel basis.

The segmentation system is applied to the DTI-datasets and the optic radiation in the two groups is identified. The left side of Fig. 1 shows the final segmented optic radiation on non-diffusion weighted axial slices with $b=0$ from two sample subjects. The color coded fractional anisotropy representation of the DTI-data is demonstrated on the right side of the figure.

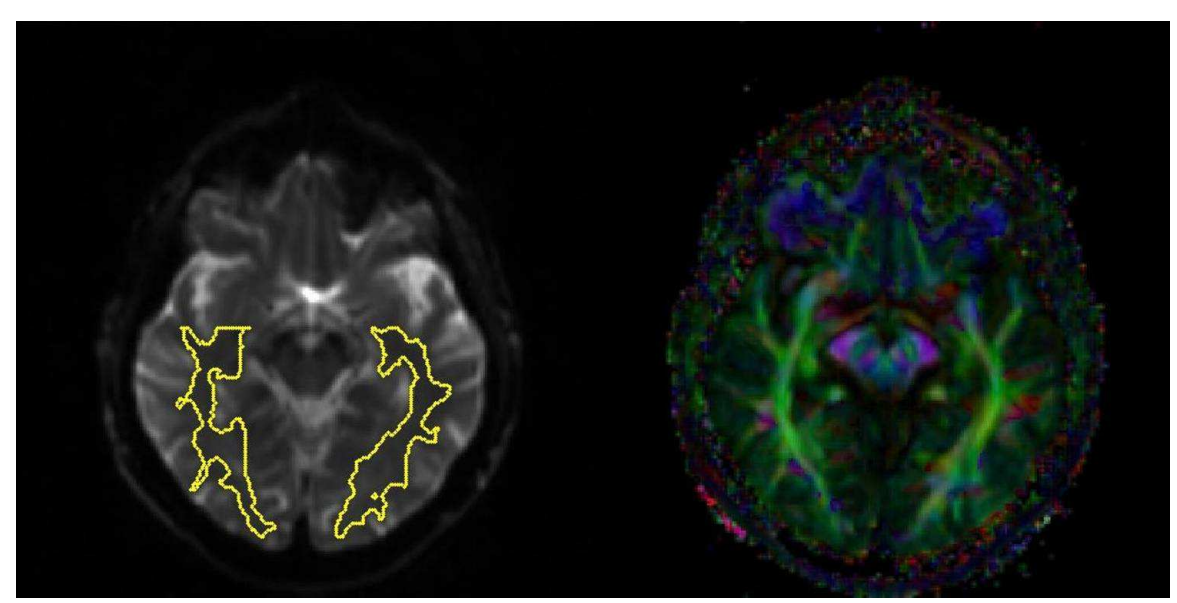

(a)

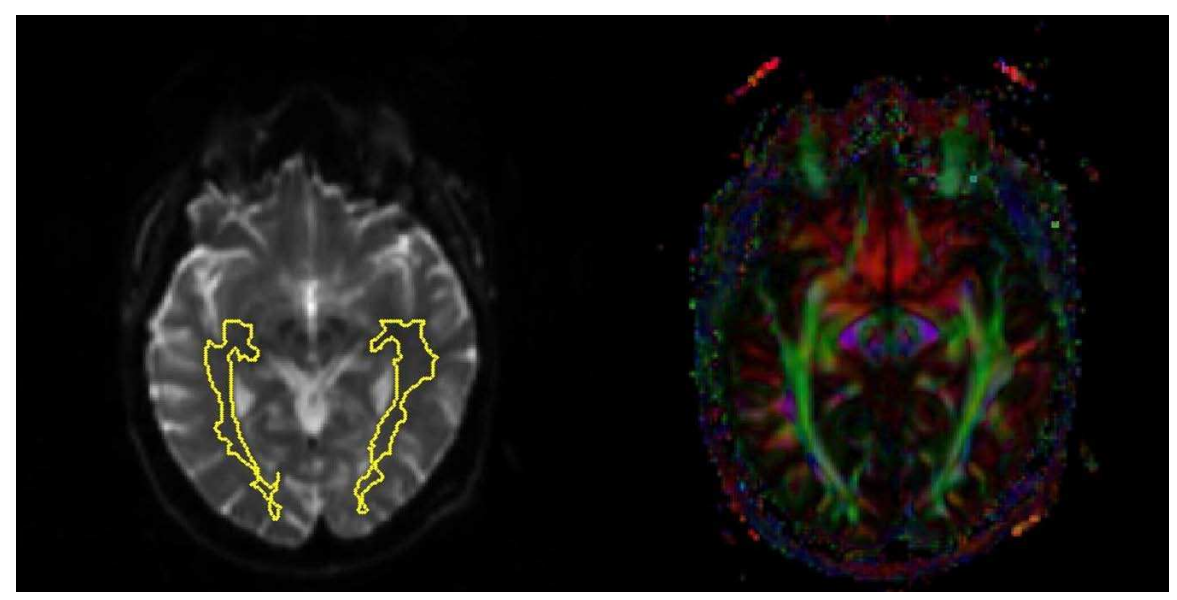

(b)

Fig. 1 Segmentation of the optic radiation in two sample subjects shown on a non-diffusion weighted image $(b=0)$ on the left side. The color coded fractional anisotropy image is shown on the right side. The main fiber bundle of the optic radiation and the lateral geniculate nucleus (LGN) of the visual pathway are clearly identified. 
The segmentation results were evaluated by comparing them with a manual segmentation of the optic radiation main fiber bundle performed by a physician experienced in neuroimaging. The accuracy of the segmentation system is calculated as the percentage of the overlap volume between the automatic segmentation results and the manual segmentation to the total volume of the manually segmented optic radiation. The segmentation accuracy is summarized in Table 1. The accuracy of the segmentation results is $82.71 \%$ for the normal subjects and $82.76 \%$ for the glaucoma group.

Table 1 The segmentation accuracy of the normal subjects and glaucoma patients

\begin{tabular}{lll}
\hline Subjects' Class & Number of Subjects & Segmentation Accuracy \\
\hline Normal Subjects & 9 & $82.71 \%$ \\
Glaucoma patients & 9 & $82.76 \%$ \\
\hline
\end{tabular}

The analysis of the segmentation errors showed that the errors typically occur in the region where the optic radiation branches in the proximity of the visual cortex. Due to the branching of the optic radiation in this region, the incoherency increases and the anterior-posterior direction is no longer the dominating diffusion direction which is the principal segmentation assumption for the proposed algorithm. Another source of errors is the relatively small coherent fiber bundles intersecting the optic radiation and sharing the anterior-posterior diffusion direction near the intersection location. Figure 2 shows the mentioned classes of errors on a sample subject as indicated by arrows.

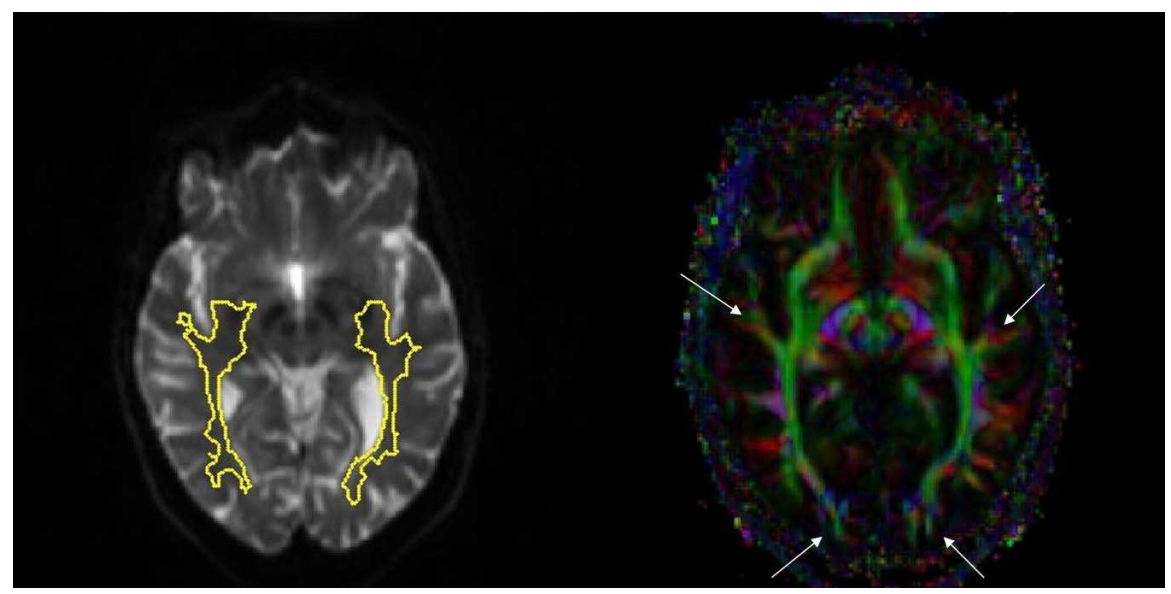

Fig. 2 The errors of segmentation of the optic radiation demonstrated on a sample subject as indicated by arrows 
The effect of glaucoma on the visual system specifically the optic nerve and the optic radiation was investigated in [13]. The correlation between glaucoma and diffusion tensor derived parameters such as fractional anisotropy and mean diffusivity was studied. The fractional anisotropy was found to be significantly lower in the glaucoma group when compared to the normal subjects. On the other hand, the mean diffusivity was significantly higher in glaucoma patients than in the normal subjects. This yields that the diffusion tensors within the optic radiation are generally affected by the presence of the neurologic pathology of glaucoma. Despite these findings, the proposed automated segmentation algorithm has approximately the same accuracy for normal subjects and glaucoma patients. This robustness is due to the dependence of the system on the physiological and anatomical properties which are slightly affected by glaucoma.

The high individual variability of the brain fiber structure and the special nature of DTI-data require great attention when dealing with the segmentation of major fiber bundles. The diffusion tensor contains information about the diffusion direction and the degree of diffusion anisotropy. So, the segmentation based on anisotropy measures or diffusion directions only results in a loss of information and inaccuracy in segmentation. Employing a Euclidean metric for measuring the similarity between diffusion tensors ignores the Riemannian nature of the tensor space and does not represent adequately the dissimilarity between tensors. Most of the proposed segmentation algorithms do not address the problem of system initialization which is usually done by a medical expert or roughly. This leads to an increased number of system iterations and the necessity for an experienced medical user. The proposed segmentation system overcomes the mentioned problems reducing the variations of the human initialization.

\section{Conclusion and Future Work}

A system has been proposed for the automatic segmentation of the optic radiation using DTI based on dissimilarity measure and the coherency property within the optic radiation fiber bundles. The automation eliminates medical-experts' intervention for identifying the optic radiation and allows the processing of large number of subjects. The system initialization problem is addressed by utilizing prior knowledge about the physiological and anatomical properties of the optic radiation to automatically provide robust estimation of the optic radiation. The incorporation of the Log-Euclidean framework in the statistical level set framework is suitable and efficient for DTI segmentation because it accounts for the Riemannian nature of the tensor space and incorporates the whole tensor information in a probabilistic framework. The system is implemented and tested using real DTI-data. The experimental results indicate that the system shows high efficiency in determining the main fiber bundle of the optic radiation for normal subjects as well as pathological subjects with glaucoma. 
The automated identification of the optic radiation will be utilized in a following study to investigate the correlation between glaucoma and the quantification of the changes occurred in the optic radiation. This aims to give further insight into the glaucoma disease and its effect on the various parts of the human visual system. The identification of the optic radiation connectivity on the visual cortex is another future goal. This requires the development of a robust tractography algorithm to be able to accurately identify the highly variable branches of the optic radiation while taking into consideration the complex fiber situations (e.g. crossing, branching, etc...) and the uncertainties in the diffusion tensor data.

Acknowledgements The authors would like to thank Dr. B. Acar from Bogazici University (www.vavlab.ee.boun.edu.tr) for the valuable discussion of the segmentation system. The authors gratefully acknowledge funding of German academic exchange service (DAAD) and the Erlangen Graduate School in Advanced Optical Technologies (SAOT) by the German National Science Foundation (DFG) in the framework of the excellence initiative.

\section{References}

1. Alexander, A.L., Hasan, K.M., Lazar, M., Tsuruda, J.S., Parker, D.L.: Analysis of partial volume effects in diffusion-tensor MRI. Magnetic Resonance in Medicine 45(5), 770-780 (2001)

2. Arsigny, V., Fillard, P., Pennec, X., Ayache, N.: Log-Euclidean metrics for fast and simple calculus on diffusion tensors. Magnetic Resonance in Medicine 56(2), 411-421 (2006)

3. Basser, P.J., Jones, D.K.: Diffusion-tensor MRI: theory, experimental design and data analysis - a technical review. NMR Biomed 15(7-8), 456-467 (2002)

4. Basser, P.J., Pajevic, S., Pierpaoli, C., Duda, J., Aldroubi, A.: In vivo fiber tractography using DT-MRI data. Magnetic Resonance in Medicine 44(4), 625-632 (2000)

5. Basser, P.J., Pierpaoli, C.: Microstructural and physiological features of tissues elucidated by quantitative-diffusion-tensor MRI. Journal of magnetic resonance, Series B 111(3), 209 - 219 (1996)

6. Bozkaya, U., Acar, B.: SMT: Split and merge tractography for DT-MRI. In: MICCAI, Brisbane, Australia. Lecture Notes in Computer Science, vol. 4792, pp. 153-160. Springer (2007)

7. Cartes-Zumelzu, F.W., Stavrou, I., Castillo, M., Eisenhuber, E., Knosp, E., Thurnher, M.M.: Diffusion-weighted imaging in the assessment of brain abscesses therapy. American Journal of Neuroradiology 25(8), 1310-1317 (2004)

8. Chen, T.F., Lin, C.C., Chen, Y.F., Liu, H.M., Hua, M.S., Huang, Y.C., Chiu, M.J.: Diffusion tensor changes in patients with amnesic mild cognitive impairment and various dementias. Psychiatry Research: Neuroimaging 173(1), 15-21 (2009)

9. Conturo, T.E., Lori, N.F., Cull, T.S., Akbudak, E., Snyder, A.Z., Shimony, J.S., Mckinstry, R.C., Burton, H., Raichle, M.E.: Tracking neuronal fiber pathways in the living human brain. Proc Natl Acad Sci U S A 96(18), 10,422-10,427 (1999)

10. Corouge, I., Fletcher, P., Joshi, S., Gouttard, S., Gerig, G.: Fiber tract-oriented statistics for quantitative diffusion tensor MRI analysis. Medical Image Analysis 10(5), 786-798 (2006)

11. Dong, Q., Welsh, R.C., Chenevert, T.L., Carlos, R.C., Maly-Sundgren, P., Gomez-Hassan, D.M., Mukherji, S.K.: Clinical applications of diffusion tensor imaging. Journal of Magnetic Resonance Imaging 19, 6-18 (2004)

12. Fletcher, P.T., Joshi, S.C.: Riemannian geometry for the statistical analysis of diffusion tensor data. Signal Processing 87(2), 250-262 (2007)

13. Garaci, F.G., Bolacchi, F., Cerulli, A., Melis, M., Span, A., Cedrone, C., Floris, R., Simonetti, G., Nucci, C.: Optic Nerve and Optic Radiation Neurodegeneration in Patients with Glaucoma: In Vivo Analysis with 3-T Diffusion-Tensor MR Imaging. Radiology 252(2), 496-501 (2009) 
14. Hamarneh, G., Hradsky, J.: DTMRI segmentation using DT-snakes and DT-livewire. In: IEEE International Symposium on Signal Processing and Information Technology, pp. 513-518 (2006)

15. Henry, R.G., Oh, J., Nelson, S.J., Pelletier, D.: Directional diffusion in relapsing-remitting multiple sclerosis: A possible in vivo signature of Wallerian degeneration. Journal of Magnetic Resonance Imaging 18(4), 420-426 (2003)

16. Hoffmann, E., Zangwill, L., Crowston, J., Weinreb, R.: Optic disk size and glaucoma. Survey of Ophthalmology 52(5), 32-49 (2007)

17. Huang, J., Friedland, R., Auchus, A.: Diffusion tensor imaging of normal-appearing white matter in mild cognitive impairment and early alzheimer disease: Preliminary evidence of axonal degeneration in the temporal lobe. American Journal of Neuroradiology 28(10), 19431948 (2007)

18. Hui, E., Fu, Q., So, K., Wu, E.: Diffusion tensor MR study of optic nerve degeneration in glaucoma. In: Engineering in Medicine and Biology Society, 2007. EMBS 2007. 29th Annual International Conference of the IEEE, pp. 4312-4315 (2007)

19. Huppi, P.S., Murphy, B., Maier, S.E., Zientara, G.P., Inder, T.E., Barnes, P.D., Kikinis, R., Jolesz, F.A., Volpe, J.J.: Microstructural brain development after perinatal cerebral white matter injury assessed by diffusion tensor magnetic resonance imaging. Pediatrics 107(3), 455$460(2001)$

20. Jones, D.: Tractography gone wild: Probabilistic fibre tracking using the wild bootstrap with diffusion tensor MRI. IEEE Transactions on Medical Imaging 27(9), 1268-1274 (2008)

21. Lansberg, M.G., Norbash, A.M., Marks, M.P., Tong, D.C., Moseley, M.E., Albers, G.W.: Advantages of adding diffusion-weighted magnetic resonance imaging to conventional magnetic resonance imaging for evaluating acute stroke. Arch Neurol 57(9), 1311-1316 (2000)

22. Lazar, M., Weinstein, D.M., Tsuruda, J.S., Hasan, K.M., Arfanakis, K., Meyerand, M.E., Badie, B., Rowley, H.A., Haughton, V., Field, A., Alexander, A.L.: White matter tractography using diffusion tensor deflection. Hum Brain Mapp 18(4), 306-321 (2003)

23. Le Bihan, D., Mangin, J., Poupon, C., Clark, C., Pappata, S., Molko, N., Chabriat, H.: Diffusion tensor imaging: concepts and applications. Journal of Magnetic Resonance Imaging 13(4), 534-546 (2001)

24. Lee, S., Uhm, K., Hong, C.: Retinal vessel diameter in normal and primary open-angle glaucoma. Korean Journal of Ophthalmology: KJO 12(1), 51-59 (1998)

25. Lenglet, C., Rousson, M., Deriche, R.: DTI segmentation by statistical surface evolution. IEEE Transactions on Medical Imaging 25(6), 685-700 (2006)

26. Mori, S., Barker, P.B.: Diffusion magnetic resonance imaging: Its principle and applications. The Anatomical Record 257(3), 102-109 (1999)

27. Mori, S., Oishi, K., Jiang, H., Jiang, L., Li, X., Akhter, K., Hua, K., Faria, A.V., Mahmood, A., Woods, R., Toga, A.W., Pike, G.B., Neto, P.R., Evans, A., Zhang, J., Huang, H., Miller, M.I., van Zijl, P., Mazziotta, J.: Stereotaxic white matter atlas based on diffusion tensor imaging in an ICBM template. Neuroimage 40(2), 570-582 (2008)

28. Moseley, M.E., Cohen, Y., Mintorovitch, J., Chileuitt, L., Shimizu, H., Kucharczyk, J., Wendland, M.F., Weinstein, P.R.: Early detection of regional cerebral ischemia in cats: Comparison of diffusion- and T2-weighted MRI and spectroscopy. Magnetic Resonance in Medicine 14(2), 330-346 (1990)

29. Nucifora, P., Verma, R., Lee, S., Melhem, E.: Diffusion-tensor MR imaging and tractography: exploring brain microstructure and connectivity. Radiology 245(2), 367-384 (2007)

30. Parker, G.J., Haroon, H.A., Wheeler-Kingshott, C.A.: A framework for a streamline-based probabilistic index of connectivity (PICo) using a structural interpretation of MRI diffusion measurements. Journal of Magnetic Resonance Imaging 18(2), 242-254 (2003)

31. Pennec, X., Fillard, P., Ayache, N.: A riemannian framework for tensor computing. Int. J. Comput. Vision 66(1), 41-66 (2006)

32. Perona, P., Malik, J.: Scale-space and edge detection using anisotropic diffusion. IEEE Transactions on Pattern Analysis and Machine Intelligence 12(7), 629-639 (1990)

33. Polo, V., Larrosa, J., Ferreras, A., Mayoral, F., Pueyo, V., Honrubia, F.: Retinal nerve fiber layer evaluation in open-angle glaucoma. Ophthalmologica 223(1), 2-6 (2009) 
34. Salat, D., Tuch, D., Greve, D., van der Kouwe, A., Hevelone, N., Zaleta, A., Rosen, B., Fischl, B., Corkin, S., Rosas, H.D., Dale, A.: Age-related alterations in white matter microstructure measured by diffusion tensor imaging. Neurobiology of Aging 26(8), 1215-1227 (2005)

35. Staempfli, P., Jaermann, T., Crelier, G., Kollias, S., Valavanis, A., Boesiger, P.: Resolving fiber crossing using advanced fast marching tractography based on diffusion tensor imaging. NeuroImage 30(1), 110 - 120 (2006)

36. Wakana, S., Jiang, H., Nagae-Poetscher, L., van Zijl, P., Mori, S.: Fiber tract-based atlas of human white matter anatomy. Radiology 230(1), 77-87 (2004)

37. Wang, Z., Vemuri, B.: DTI segmentation using an information theoretic tensor dissimilarity measure. IEEE Transactions on Medical Imaging 24(10), 1267-1277 (2005)

38. Yörük, E., Acar, B., Bammer, R.: A physical model for MR-DTI based connectivity map computation. In: MICCAI, Palm Springs, CA. Lecture Notes in Computer Science, vol. 3749, pp. 213-220. Springer (2005)

39. Zhou, X.: Diffusion tensor imaging: techniques and clinical applications. Engineering in Medicine and Biology Society, 2004. IEMBS '04. 26th Annual International Conference of the IEEE 2, 5223-5225 (2004)

40. Zhukov, L., Museth, K., Breen, D., Whitaker, R., Barr, A.: Level set modeling and segmentation of DT-MRI brain data. Journal of Electronic Imaging 12(1), 125-133 (2003) 